\title{
AN EXTENSION OF A RESULT OF ANDJEL
}

\author{
By TOM MOUNTFORD \\ University of California, Los Angeles
}

\begin{abstract}
We prove, using results for hydrodynamic limits, that an exclusion process starting from an ergodic initial distribution converges to product measure in one dimension. Our only assumption is the existence of a nonzero mean for the underlying random walk.
\end{abstract}

1. Introduction. In this note we consider a class of interacting particle systems closely related to systems of queues in tandem.

Classically interacting particle systems are Markov processes $\eta_{t}$ on state space $\{0,1\}^{\mathbb{Z}^{d}}$. Then $\eta_{t}(x)=1$ is interpreted to mean that there is a particle at site $x$ at time $t$. Necessarily, there can be at most one particle per site at any given time $t$. The particles move or are created or destroyed at rates depending on the state of the configuration $\eta_{t}$ locally. In this paper we are interested in the exclusion process; this is a process where particles are neither created nor destroyed. Each particle tries to move after waiting exponential amounts of time. After such times a new site is selected according to some fixed (for the process) random walk kernel. If at this time the selected site is already occupied by a particle, the move is suppressed and the first particle stays where it originally was; otherwise it does move to the new site.

We restrict attention to the one-dimensional exclusion process. This is an interacting particle system on $\{0,1\}^{\mathbb{Z}}$ with generator

$$
\Omega f(\xi)=\sum_{x, y}\left(f\left(\xi^{x y}\right)-f(\xi)\right) p(y-x) \xi(x)(1-\xi(y)),
$$

where

$$
\begin{aligned}
& \xi^{x y}(z)=\xi(z) \quad \text { for } z \neq x \text { or } y, \\
& \xi^{x y}(x)=\xi(y), \quad \xi^{x y}(y)=\xi(x)
\end{aligned}
$$

and where $p($ ) is a probability distribution on the integers.

The exclusion process and tandem systems of queues are intimately related. The connection has long been recognized (see [6]). There is an explicit mapping of the totally asymmetric nearest neighbor exclusion process [where $p(1)=1$ ] to the queuing system consisting of a sequence of independent identically distributed memoryless servers and customers passing through from one queue to the succeeding queue: one identifies particles with customers and vacancies with servers. Given a vacancy, if its left neighbor is also vacant then it

Received July 1999; revised March 2000. AMS 2000 subject classifications. $60 \mathrm{~K} 35$.

Key words and phrases. Exclusion process, convergence in distribution. 
is empty; otherwise all 1's between the vacancy and the next vacancy to the left are regarded as customers queuing up for service at the first vacancy. The particle immediately to the left of the vacancy will be currently receiving service. One can therefore simply transport results from one set-up to the other. Another connection (at a heursitic level) is that both the exclusion process and queueing models possess a conservation property: particles or customers are neither created nor destroyed. This common property and similar coupling arguments enable one to prove similar results for stationary translation invariant processes or arrival processes for queues. One has the heuristic that what holds for one system holds for the other. This led to an argument of [2] for convergence in a translation invariant conservative particle system being adapted in [10] to give a convergence result for a stationary, ergodic arrival process that passed through a sequence of memoryless queues. It is our hope that the approach detailed in this paper can give results for queuing systems in tandem.

Though it is a bit artificial, one could think of our exclusion process as representing a queuing system where particles are customers and sites represent servers. When customers are served (after an exponential amount of time), they select a new server according to kernel $p($ ). If the server chosen is currently working, then customers engage in a new service with their original servers.

For technical reasons we will assume in this paper that

$$
\begin{aligned}
& \sum_{n \geq 0} p^{n}(z)+p^{n}(-z)>0 \quad \forall z \in \mathbb{Z}, \\
& \sum_{x}|x| p(x)<\infty \\
& \sum x p(x) \neq 0 .
\end{aligned}
$$

In fact, w.l.o.g., we suppose

$$
\sum x p(x)=m>0 .
$$

For a full account see [8], for an account of recent developments see [9] and for a rich account of "hydrodynamic limits" see [7].

For each $\alpha \in[0,1], \rho_{\alpha}$ (product $\alpha$-Bernoulli measure) is invariant for the process and (see [8]) it can be shown that there are no other extremal invariant distributions for the process that are stationary under translations (which is also the case for arrival processes to a $\cdot / M / 1$ queue).

It is natural to conjecture that if $\xi_{0}$ has a distribution that is stationary under translations and of nonconstant density $\alpha$, then $\xi_{t} \longrightarrow^{D} \rho_{\alpha}$ as $t$ tends to infinity.

In generality all that is known is that

$$
\xi_{t} \stackrel{D}{\longrightarrow} \int_{0}^{1} \lambda(d \alpha) \rho_{\alpha}
$$


a mixture of product measures. If $p$ is symmetric (and irreducible) then we have indeed that $\xi_{t} \rightarrow^{D} \rho_{\alpha}$ by self-duality (see [8]).

The first result for nonstationary $p$ is [1] where it is proved that in the nearest neighbor case with $d=1$, we have the appropriate convergence. Recently [2] showed that for finite range $p$ (again in $d=1$ ) we have convergence to product measure.

Our result is the following theorem.

THEOREM. If $\xi_{0}$ is stationary under translations with

$$
\frac{1}{2 n+1} \sum_{|x| \leq n} \xi_{0}(x) \stackrel{\text { a.s. }}{\longrightarrow} \alpha \in[0,1]
$$

and $p$ satisfies (I), (II), (III) then

$$
\xi_{t} \stackrel{D}{\longrightarrow} \rho_{\alpha}
$$

It should be noted that since we assume that $\Sigma x p(x) \neq 0$, our result is not an extension of that of [2].

The key ideas are the results of [11] and various coupling ideas. In Section 2 we simply note some "irreducibility" properties. In Section 3 we consider Rezakhanlou's work [11] and consequences for particle systems with "first" and "second" class systems. In Section 4 we give the overall argument and the theorem's proof is completed in Section 5 .

In the remainder of the introduction we detail simple consequences of the natural coupling.

We assume that we are given a Harris system generating all processes on $\{0,1\}^{\mathbb{Z}}$. This system consists of an independent Poisson process $N^{x, y}, x, y \in$ $\mathbb{Z}$ of rate $p(y-x)$. An exclusion process is constructed from the $(N)$ 's by stipulating that at $t \in N^{x, y}$ "a particle tries to move from site $x$ to site $y$." That is, for $t \in N^{x, y}$, if immediately before time $t$,

$$
\xi(x)=1, \quad \xi(y)=0 \quad \Longrightarrow \quad \xi_{t}(x)=0, \quad \xi_{t}(y)=1 .
$$

Otherwise there is no change in process $\xi$ at time $t$.

A natural way to show that $\xi_{t}$ converges to $\rho_{\alpha}$ in distribution as $t \longrightarrow \infty$ is via coupling. We introduce process $\xi_{t}^{\alpha}$ with $\xi_{0}^{\alpha} \sim \rho_{\alpha}$ (and so $\xi_{t}^{\alpha} \sim \rho_{\alpha} \forall t$ ) and generated by the same system of Poisson processes as $\xi$. If we could show that $\xi$ and $\xi^{\alpha}$ couple in the sense that $\forall x P\left(\xi_{t}(x)=\xi_{t}^{\alpha}(x)\right) \longrightarrow 1$ as $t \longrightarrow \infty$ then we would have established the desired convergence.

A useful idea extensively exploited in, for example, [5] is to use first and second class particles. We consider a process $\xi_{t}$ on $\{0,1,2\}^{\mathbb{Z}}$ [again generated by $\left.\left(N^{x, y}\right)_{x, y \in \mathbb{Z}}\right]$ so that at time $t \in N^{x, y}$. If immediately before $t$,

$$
\begin{aligned}
& \xi(x)=1, \quad \xi(y)=j \quad \text { then } \quad \xi_{t}(x)=j, \quad \xi_{t}(y)=1, \\
& \xi(x)=2, \quad \xi(y)=j \neq 1 \quad \text { then } \quad \xi_{t}(x)=j, \quad \xi_{t}(y)=2 .
\end{aligned}
$$


Otherwise $\xi$ is unchanged at time $t$.

This is easily seen if we consider either

$$
\eta_{t}(x)=1 \quad \Longleftrightarrow \quad \xi_{t}(x)=1
$$

or

$$
\eta_{t}(x)=1 \quad \Longleftrightarrow \quad \xi_{t}(x) \neq 0 .
$$

Then we have the usual exclusion processes corresponding to $p$. One can basically have as many classes of particles as desired. We (as a limit) introduce a process $\eta_{t}$ on $[0,1]^{\mathbb{Z}}$ with generator

$$
\Omega f(\eta)=\sum_{x, y}\left(f\left(\eta^{x, y}\right)-f(\eta)\right) p(y-x) I_{\eta(x) \leq \eta(y)},
$$

where (as before)

$$
\begin{aligned}
& \eta^{x, y}(z)=\eta(z) \quad \text { for } \quad z \neq x, y, \\
& \eta^{x, y}(x)=\eta(y), \quad \eta^{x, y}(y)=\eta(x)
\end{aligned}
$$

and we fix $\left(\eta_{0}(x)\right)_{x \in \mathbb{Z}}$ to be iid $U[0,1]$ r.v's. Then $\forall \beta \in[0,1]$ if we consider

$$
\xi_{t}^{\beta}(x)=1 \quad \Longleftrightarrow \quad \eta_{t}(x) \leq \beta .
$$

Then $\xi^{\beta}$ is an exclusion process starting in $\rho_{\beta}$.

Furthermore, if for $\beta<\gamma$,

$$
\begin{array}{ll}
\xi_{t}^{\beta, \gamma}(x)=1 & \Longleftrightarrow \eta_{t}(x) \leq \beta, \\
\xi_{t}^{\beta, \gamma}(x)=2 \quad \Longleftrightarrow \quad \eta_{t}(x) \in(\beta, \gamma), \\
\xi_{t}^{\beta, \gamma}(x)=0 \quad \text { otherwise. }
\end{array}
$$

Then $\xi^{\beta, \gamma}$ is an exclusion process of first and second class particles.

If $\eta$ is generated by the same Harris system as our general exclusion process $\xi_{t}$, then, as already noted,

$$
\xi_{t} \longrightarrow \rho_{\alpha} \quad \text { if } \quad \forall x P\left(\xi_{t}(x)=\xi_{t}^{\alpha}(x)\right) \longrightarrow 1 .
$$

In our coupling of $\left(\xi_{t}, \eta_{t}\right)$ we suppose $\eta_{0}$ is independent of $\xi_{0}$ and (wlog) that $\xi_{0}$ is ergodic so that $\forall t\left(\xi_{t}, \eta_{t}\right)$ is ergodic. We define

$$
\mu_{t}[0, c]=\lim _{n \rightarrow \infty} \frac{1}{2 \alpha n} \sum_{|x| \leq n} I_{\xi_{t}(x)=1} I_{\eta_{t}(x) \leq c}
$$


We can label the $\xi$-particles so that a particle only moves from site $x$ to site $y$ at times $t \in N^{x y}$ or $N^{y x}$ and so that the associated $\eta$-value for a $\xi$-particle never increases. Then the quantity

$$
\frac{1}{2 \alpha n} \sum_{|x| \leq n} I_{\xi_{t}(x)=1} I_{\eta_{t}(x) \leq c}
$$

is equal to a nondecreasing process plus "edge effects" resulting from particles moving in and out of spatial interval $[-n, n]$. As $n$ becomes large these "edge effects become negligible." Thus we have (simultaneously over all $c \in[0,1]$ ) that $\mu_{t}[0, c]$ increases in time. It is easy to see that we have

$$
\mu_{t}[0, c] \uparrow \mu_{\infty}[0, c] \text { for } \mu_{\infty} \text { a measure on }[0,1] .
$$

As noted if $\mu_{\infty}$ is $U[0, \alpha]$ (the uniform distribution on $[0, \alpha]$ ) then we have that asymptotically all $\xi$-particles are coalesced with $\eta$-particles of value in $[0, \alpha]$ and vice versa. That is, $\xi_{t} \longrightarrow^{D} \rho_{\alpha}$. So in Section 2 we will begin an argument by contradiction and suppose $\mu_{\infty} \neq U[0, \alpha]$.

2. Coupling foundations. We begin this section with a basic result concerning two finite exclusion processes generated by the same Harris system.

An exclusion process on $\{0,1\}^{I}$ for finite interval $I$ is the Markov process with generator

$$
\Omega f(\xi)=\sum_{x, y \in I}\left(f\left(\xi^{x y}\right)-f(\xi)\right) p(y-x) \xi(x)(1-\xi(y)) .
$$

We denote exclusion processes on interval $[0, m]$ by $\xi^{m}, \eta^{m}$. We say two such processes $\eta^{m}$ and $\xi^{m}$ are coupled if they are generated by the same Harris sytem.

LEMMA 2.1. Under the assumptions for $p(\cdot)$, there exist an $m_{0}$ so that for $m \geq m_{0}$, if $\xi^{m}, \eta^{m}$ are coupled exclusion processes on $\{0,1\}^{[0, m]}$,

$$
\begin{gathered}
\xi_{0}^{m}(0)=1=1-\eta_{0}^{m}(0), \\
\xi_{0}^{m}(m)=0=1-\eta_{0}^{m}(m),
\end{gathered}
$$

then

$$
P\left(\sum_{i=0}^{m}\left|\xi_{0}^{m}(i)-\eta_{1}^{m}(i)\right| \leq \sum_{i=0}^{m}\left|\xi_{0}^{m}(i)-\eta_{0}^{m}(i)\right|-2\right)>0 .
$$

REMARK. In other words, the lemma asserts that there is a strictly positive chance that an uncoupled $\xi^{m}$-particle will couple with an uncoupled $\eta^{m}$-particle if there exist two such particles sufficiently far apart.

Proof of Lemma 2.1. It is easy to see that for $m$ sufficiently large there will exist a random walk path $0=x_{0}, x_{1}, x_{2}, \ldots, x_{R}=m$ contained in $[0, m]$. Here a random walk path satisfies $p\left(x_{i}-x_{i-1}\right)>0 \forall i$. If $R=1$, then trivally there is a strictly positive chance that the $\xi^{m}$-particle at 0 jumps to site $m$ 
(thereby coupling with the $\eta^{m}$-particle at $m$ ) before any other jump. So we suppose $R>1$, use induction on $R$ and suppose that whenever (for whatever $m$ ) there exists a random walk path of length $R-1$ or less from an uncoupled $\xi$-particle to an uncoupled $\eta$-particle, a coupling of these two particles may occur.

First suppose that for some $0<j<R$ we have $\xi_{0}^{m}\left(x_{j}\right) \neq \eta_{0}^{m}\left(x_{j}\right)$. If $\xi_{0}^{m}\left(x_{j}\right)=1=1-\eta_{0}^{m}\left(x_{j}\right)$ then $x_{j}, x_{j+1} \cdots x_{R}$ is a path of length shorter than $R$ from an uncoupled $\xi^{m}$-particle to an uncoupled $\eta^{m}$-particle and so the result follows by induction. Similarly, if $\xi_{0}^{m}\left(x_{j}\right)=0=1-\eta_{0}^{m}\left(x_{j}\right)$, then $x_{0}, x_{1}, \ldots, x_{j}$ is such a path.

So we assume $\xi_{0}^{m}\left(x_{i}\right)=\eta_{0}^{m}\left(x_{i}\right) \forall 0<i<R$.

Let $j=\inf \left\{i: \xi_{0}^{m}\left(x_{i}\right)=0\right\}$; in this case with positive probability $\left(\xi^{m}, \eta^{m}\right)$ moves to $\left(\xi^{m, 1}, \eta^{m, 1}\right)\left(\xi^{m, 2}, \eta^{m, 2}\right) \cdots\left(\xi^{m, j}, \eta^{m, j}\right)$ where $\left(\xi^{m, k}, \eta^{m, k}\right)(y)=$ $\left(\xi_{0}^{m}, \eta_{0}^{m}\right)(y)$ for $y \notin\left\{x_{0}, x_{1}, \ldots x_{j}\right\}$ and for $0<k<j$,

$$
\begin{aligned}
\left(\xi^{m, k}, \eta^{m, k}\right)\left(x_{j-k}\right) & =\left(\xi_{0}^{m}, \eta_{0}^{m}\right)\left(x_{j}\right), \\
\left(\xi^{m, k}, \eta^{m, k}\right)\left(x_{r}\right) & =(1,1) \quad \text { for } \quad r>j-k, \\
\left(\xi^{m, k}, \eta^{m, k}\right)\left(x_{r}\right) & =\left(\xi_{0}^{m}, \eta_{0}^{m}\right)\left(x_{r}\right), \quad r<j-k
\end{aligned}
$$

and $\left(\xi^{m, j}, \eta^{m, j}\right)$ is obtained from $\left(\xi^{m, j-1}, \eta^{m, j-1}\right)$ by the $\xi$-particle at 0 moving to site $x_{i}$. If $x_{j}=x_{R}=m$ then this move delivers the coupling. On the other hand, if $x_{j} \neq x_{R}$ then for configuration $\left(\xi^{m, j}, \eta^{m, j}\right) x_{1}, x_{2}, \ldots x_{R}$ is a path of length $R-1$, from an uncoupled $\xi$-particle to an uncoupled $\eta$-particle.

For two coupled processes $\eta, \xi$ on an interval $I$, the quantity $\sum_{I} \mid \xi_{t}(x)-$ $\eta_{t}(x) \mid$ cannot increase at any time $t$. If this quantity strictly decreases at time $t$, we say that a $\xi$-particle has joined an $\eta$-particle.

We return to the coupled process $\left(\xi_{t}, \eta_{t}\right)_{t \geq 0}$. Here $\xi_{t}$ is our exclusion process starting from translation invariant distribution of (nonrandom) density $\alpha$ and $\eta_{t}$ is our process on $[0,1]^{\mathbb{Z}}$.

If the associated measures $\left(\mu_{t}=\operatorname{disn} \eta_{t}(0) \mid \xi_{t}(0)=1\right)$ satisfy $\mu_{t} \rightarrow^{D}$ $U[0, \alpha]$, then we have $\xi_{t} \rightarrow{ }^{D} \rho_{\alpha}$. We therefore suppose that $\mu_{t} \nrightarrow \rightarrow U[0, \alpha]$. In this case we must have the existence of $\varepsilon, \delta>0$ and $0<a<\alpha-4 \delta<\alpha+4 \delta<$ $b<b+\delta<1$, so that

$$
\begin{aligned}
& \mu_{\infty}([a, a+\delta]) \leq \frac{\delta}{\alpha}(1-\varepsilon), \\
& \mu_{\infty}([b, b+\delta]) \geq \frac{\delta \varepsilon}{\alpha} .
\end{aligned}
$$

Fix such $\varepsilon, \delta, a$ and $b$. These quantities are given to us by our assumption of nonconvergence. The quantities $\varepsilon, \delta$ can be thought of as small but need not be so in principle. We will now introduce $\varepsilon^{\prime}$ (and later $\gamma$ ) to be numbers which are very small in comparison to $\varepsilon \delta$. The large number $N$ will be chosen so that various laws of large numbers hold (to within some multiple of this $\varepsilon^{\prime}$ and $\gamma^{\prime}$ ). 
Choose $\varepsilon^{\prime} \ll(\varepsilon \delta)^{3} \wedge \frac{\varepsilon \delta}{1800\left(1+\sum|x| p(x)\right)}$ (we will introduce other upper bounds later). There exist $T\left(=T\left(\varepsilon^{\prime}\right)\right)$ so that $\forall c \in\{a, a+\delta, b, b+\delta\}$; the density of $\xi$ particles having $\eta$-label $\leq c$ does not increase by more than $\varepsilon^{\prime} / 4$ for $t \geq T$.

Now we collapse $\eta_{t}$ onto a system of particles with five classes $\eta_{t}^{i}, i=$ $1,2, \ldots, 5$, where

$$
\begin{aligned}
& \eta_{t}^{1}(x)=1 \Longleftrightarrow \eta_{t}(x) \leq a, \\
& \eta_{t}^{2}(x)=1 \quad \Longleftrightarrow \quad \eta_{t}(x) \in(a, a+\delta], \\
& \eta_{t}^{3}(x)=1 \quad \Longleftrightarrow \quad \eta_{t}(x) \in(a+\delta, b], \\
& \eta_{t}^{4}(x)=1 \quad \Longleftrightarrow \quad \eta_{t}(x) \in(b, b+\delta], \\
& \eta_{t}^{5}(x)=1 \quad \Longleftrightarrow \quad \eta_{t}(x)>b+\delta .
\end{aligned}
$$

Thus each $\xi_{t}$-particle (or vacancy) has an associated $\eta$-class in $\{1,2,3,4,5\}$. Our choice of $a, b, \varepsilon, \delta$ and $T$ ensure that $\forall t \geq T$, the density of $\xi_{t}$ vacancies of $\eta$-class 2 is at least $\varepsilon \delta-\varepsilon^{\prime} / 4$, and that the density of $\xi_{t}$-particles of $\eta$-class 4 is at least $\delta \varepsilon-\varepsilon^{\prime} / 4$.

Thus we have the following lemma.

Lemma 2.2. For $N$ sufficiently large,

$$
\begin{gathered}
\sum_{x=0}^{N}\left(1-\xi_{T}(x)\right) \eta_{T}^{2}(x) \geq N\left(\varepsilon \delta-\varepsilon^{\prime}\right) \equiv M, \\
\sum_{x=N+m_{0}}^{2 N} \xi_{T}(x) \eta_{T}^{4}(x) \geq N\left(\varepsilon \delta-\varepsilon^{\prime}\right) \equiv M,
\end{gathered}
$$

outside of probability $\varepsilon^{2} / 10^{10}$.

In other words, Lemma 2.2 is asserting the existence of many $\eta$-type 2 $\xi$-vacancies and many $\eta$-type $4 \xi$-particles outside of very small probability.

Labeling. In the last two sections the arguments will turn on the behavior of $\xi$-particles and $\xi$-vacancies. We introduce a labeling of these particles and vacancies. We first stipulate that the labels should move so that the $\eta$-class of a $\xi$-vacancy does not decrease; the $\eta$-class of a $\xi$-particle does not increase.

Thus if $u$ and $v$ are $\xi$-particles at sites $x, y$, respectively, immediately before $t$ and immediately before $t, \eta^{i}(x)=1, \eta^{j}(y)=1$ for $i<j$, then if $t \in N^{x, y}$, at time $t$ label $u$ moves from site $x$ to site $y$ while label $v$ moves from site $y$ to site $x$. Thus labels can move from site $x$ to site $y$ even though the $\xi$ process is unchanged at these sites.

In time interval [ $T, k N]$ ( $k$ to be fixed later) we will introduce a labeling scheme for $\eta$ particles that will respect the priorities. We will subdivide priority (or class 2) so that $\eta$-class 2 particles in spatial interval $[0, N]$ at time $T$ 
will be assigned a new class $2^{*} ; \eta$-class $\eta$-4 particles in $\left[N+m_{0}, 2 N\right]$ at time $T$ will be assigned a new class $4^{*}$. The priority scheme will be

1 over 2 over $2^{*}$ over 3 over $4^{*}$ over 4 over 5 .

It should be noted that while the labeling of particles will thus differ from previous labelings, the overall coupled processes are the same. Of course as far as our original process is concerned, $\eta$-class $2^{*}$ particles are simply $\eta$-class 2 particles, similarly for $\eta$-class $4^{*}$ particles.

Assuming the conclusions of Lemma 2.2, let us label $M \xi$-vacancies of $\eta$-class $2^{*}$ in $[0, N]$ at time $T, i_{1}, i_{2}, \ldots, i_{M}$. Let us label $M \xi$-particles of $\eta$-class $4^{*} j_{1}, \ldots, j_{M}$. We now describe the motion of labels $i_{h}, j_{l}$.

Let $x_{t}\left(i_{h}\right)$ denote the position of $\xi$-vacancy $i_{h}$ at time $t$. If $\eta$-particle $u$ (u of whatever class) at time $t$ is at site $w=x_{t}\left(i_{h}\right)$, we say that $u$ is associated with $i_{h}$ at time $t$ (and vice versa).

First we detail the motion of $i_{h}$ if $x_{t-}\left(i_{h}\right)=w$ and $i_{h}$ is associated with a class $\ell \eta$-particle at $t$ - and $t \in N^{w, y}$.

If at $t$ - the $\eta$-class of site $y$ is $\leq \ell$ then nothing happens to label $i_{h}$ (it remains at $w$ ). If the $\eta$-class at $y$ greater than $\ell$, then $i_{h}$ moves to $y$ at time $t$ if and only if $\xi_{t-}(y)=0$. Otherwise $i_{h}$ stays at $w$ and its $\eta$-class increases.

Next, if $x_{t-}\left(i_{h}\right)=w$ and $i_{h}$ is associated with a class $\ell \eta$-particle $(\ell \in$ $\left.\left\{1,2,2^{*}, 3,4^{*}, 4,5\right\}\right)$ and $t \in N^{y, w}$ then if (at $t-$ ) the $\eta$-class of site $y$ is less than $\ell, i_{h}$ moves to site $y$ (and remains associated to the same $\eta$-particle). If the $\eta$-class of site $y$ is greater than or equal to $\ell$ then $i_{h}$ moves to $y$ if and only if $\xi_{t-}(y)=1$. In this case the $\eta$-class of $i_{h}$ may increase.

The motion of $\xi_{t}$-particles $j_{l}$ is similarly determined. The important fact is that:

(*) For $\xi$-vacancies $i_{h}$ the class of the associated $\eta$-particle never decreases.

(**) For $\xi$-particles $j_{l}$ the class of associated $\eta$-particle never increases.

3. A result of Rezakhanlou. The key tool of this paper is a result of Rezakhanlou [11]; for our purposes (though much more is proved in his paper) his theorem is as follows.

THEOREM. Let $f$ be a positive function that is bounded by 1 and continuous except at finitely many points. Let $\eta_{t}^{N}$ be a sequence of exclusion processes on $\mathbb{Z} / N$ with $\left(\eta_{0}^{N}(x)\right)$ independent Bernoulli, $P\left(\eta_{0}^{N}(x)=1\right)=f(x)$ for $x \in \mathbb{Z} / N$. Then for interal I we have $\forall \gamma>0$,

$$
P\left(\left|\frac{1}{N} \sum_{x \in(\mathbb{Z} / N) \cap I} \eta_{N t}^{N}(x)-\int_{I} u(t, x) d x\right|>\gamma\right) \longrightarrow 0 \quad \text { as } N \longrightarrow \infty,
$$

where $u$ is the unique entropy solution to

$$
\frac{\partial u}{\partial t}+m \frac{\partial(u(1-u))}{\partial x}=0, \quad u(0, x)=f(x) .
$$

[Note that $\eta^{N}$ has particles that try to jump from $x$ to $y$ at rate $p(N(y-x))$.] 
REMARK. [11] actually only explicitly treats the case where $p$ is finite range (but otherwise assumptions I-III hold). However, in $d=1$, the arguments carry over to our case without much trouble.

We consider this theorem first for $f_{1}$ and $f_{2}$ with $f_{1} \equiv a+\delta$ and $f_{2} \equiv a+\delta$ on $[0,1]^{c} \equiv a$ on $[0,1]$. If $u_{1}, u_{2}$ are the corresponding solutions to Burger's equation with initial $f_{1}$ and $f_{2}$, then

$$
u_{1} \geq u_{2} \forall t, x \text { and } \int\left(u_{1}-u_{2}\right) d x
$$

is constant in $t$ (conservation property). However (see, e.g., [7]), $u_{1}=u_{2}$ on $[m(1-2 a-2 \delta) t, 1+m(1-2 a-\delta) t]^{c}$.

Now by a very elementary argument we have that the number of particles in space interval $[0, N]$ at time 0 but not in $[0, N]$ at time $T$ or not in $[0, N]$ at time 0 but in $[0, N]$ at time $T$ is very small compared to $N$ with very large probability. Thus we have the following.

Proposition 3.1. As $N \longrightarrow \infty$ we have $\forall \gamma>0, P$ [number of $2^{*}$ particles outside $[m N(1-2 a-2 \delta) t, N(1+m(1-2 a-\delta)) t]$ at time $N t \geq N \gamma] \longrightarrow 0$.

Similarly we have $u_{2} \equiv b$, taking Burger's equation solutions $u_{i}$ with $u_{1}$ initially equal to $b$ on $[1,2]^{c}$ and equal to $b+\delta$ on $[1,2]$.

Proposition 3.2. As $N \longrightarrow \infty$ we have $\forall \gamma>0, P$ [number of $4^{*}$ particles outside $[(1+m t(1-2 b-\delta)) N,(2+m t(1-2 b)) N]$ at time $N t \geq N \gamma] \longrightarrow 0$.

Taken together we have the following proposition.

Proposition 3.3. Fix $k>\frac{1}{m(b-a-\delta)}$, then $\forall \gamma>0$ fixed we have $P$ [all but $\gamma N 2^{*}$ particles are to the right of all but $\gamma N 4^{*}$ particles at time $\left.k N\right]>1-\gamma$ for $N$ sufficiently large.

In addition to previous demands on $N$ we assume $N$ is sufficiently large that Proposition 3.3 holds with $\gamma=\left(\varepsilon^{\prime}\right)^{2} / 10^{10}$.

4. Statement of Proof. Consider a $\xi$-vacancy that is at time $T$ associated with a $\eta$-type $2^{*}$ particle $i_{h}$ (necessarily at a site on $[0, N]$ at time $T$ ).

If this $\xi$-vacancy remains associated to a $\eta$-type $2^{*}$ particle (not necessarily the same one) throughout $[T, k N]$ then (with overwhelming probability) it should (by Proposition 3.3) have had to pass over many $\xi$-particles associated with a $\eta$-type $4^{*}$ particle. From our coupling, the rate at which a $\xi$-vacancy moves from site $x$ to site $y$ is bounded by $p(y-x)+p(x-y)$. [What it actually is depends on the $(\xi, \eta)$ configuration.]

Thus we have that $N$ can be chosen so large that $\forall 1 \leq h \leq M$,

$$
P\left[i_{h} \text { leaves interval }[-2 C N, 2 C N] \text { in time } k N\right]
$$

$$
\leq\left(\varepsilon^{\prime}\right)^{3} \text { for } C=3 k\left(1+\sum|x| p(x)\right),
$$


where the bound is independent of $(\xi, \eta)_{T}$, similarly for $\xi$-particles $j_{l}, 1 \leq$ $l \leq M$.

Let $m_{0}$ be the constant of Lemma 2.1. We can choose $m_{1}$ so that $\Sigma_{|x| \geq m_{1}}(|x|+$ $\left.m_{0}\right) p(x) \leq\left(\varepsilon^{\prime}\right)^{2} /(k+1)$.

Now for $\xi$-vacancy $i_{h}$ we say an $m_{1}$ jump occurs at time $t$ if $x_{t}\left(i_{h}\right)-x_{t-}\left(i_{h}\right) \geq$ $m_{1}$. Let

$$
X\left(i_{h}\right)=\sum_{m_{1} \text { jumps } t \leq k N}\left(x_{t}\left(i_{h}\right)-x_{t-}\left(i_{h}\right)+m_{0}\right) .
$$

We similarly say for $\xi$-particle $j_{l}$ that an $m_{1}$ jump occurs if $x_{t}\left(j_{l}\right)-$ $x_{t-}\left(j_{l}\right) \leq-m_{1}$.

Let $X\left(j_{l}\right)=\Sigma_{m_{1} \text { jump at } t<k N}\left(m_{0}+x_{t-}\left(j_{l}\right)-x_{t}\left(j_{l}\right)\right)$. By the law of large numbers we can choose $N$ so large that

$$
\begin{aligned}
& \forall 1 \leq h, l \leq M \\
& \quad P\left(X\left(i_{h}\right) \geq\left(\varepsilon^{\prime}\right)^{3 / 2} N\right) \leq\left(\varepsilon^{\prime}\right)^{2}, \\
& P\left(X\left(j_{l}\right) \geq\left(\varepsilon^{\prime}\right)^{3 / 2} N\right) \leq\left(\varepsilon^{\prime}\right)^{2} .
\end{aligned}
$$

The idea of our proof is that, with large probability by time $k N$, either a reasonable number of the $\xi$-vacancies of $\eta$-type $2^{*}$ are now associated with higher $\eta$-types or a reasonable number of $\xi$-particles of $\eta$-type $4^{*}$ are now associated with lower $\eta$-types [and by assumption (D) this will have taken place inside $[-2 C N, 2 C N]$ ], or (by Proposition 3.3) a large number of $\xi$-vacancies of $\eta$-type $2^{*}$ have passed by a large number of $\xi$-particles of $\eta$-type $4^{*}$.

However, the former case is forbidden by our choice of $T$. For the latter, stipulation (E) on the size of $N$ means that, outside of small probability, most $\xi$-vacancies will pass close to most $\xi$-particles while the former travel to the right of the latter, outside of very small probability.

5. Completion of proof. An $\xi$-vacancy $i_{h}$ can pass $\xi$-particle $j_{l}$ in three ways:

1. For some $t \subset[T, k N], x_{t}\left(j_{\ell}\right) \in\left[x_{t}\left(i_{h}\right)+m_{0}, x_{t}\left(i_{h}\right)+m_{1}+m_{0}\right]$;

2. For some $t \in[T, k N], x_{t}\left(i_{h}\right)+m_{0}>x_{t}\left(j_{\ell}\right)>x_{t-}\left(i_{h}\right)+m_{1}+m_{0}$;

3. For some $t \in[T, k N], x_{t}\left(j_{\ell}\right)-m_{0}<x_{t}\left(i_{h}\right)<x_{t-}\left(j_{\ell}\right)-\left(m_{1}+m_{0}\right)$.

Assumptions (E) on the size of $N$ imply that the expected number of $j_{\ell}$ passed in manner (2) or (3) is likely to be small. So it remains to consider the possibility of many $j_{\ell}$ passing by $i_{h}$ after coming "within range." By Lemma 2.1 if $j_{\ell}$ is in $\left[x_{t}\left(i_{h}\right)+m_{0}, x_{t}\left(i_{h}\right)+m_{1}+m_{0}\right]$ then (if both have their original $\eta$-class) there is a reasonable chance of some coupling taking place. A problem (to be addressed via "windows") is that the coupling between $\eta-2^{*}$-particles and $\xi$ particles in $\left[x_{t}\left(i_{h}\right)+m_{0}, x_{t}\left(i_{h}\right)+m_{1}+m_{0}\right]$ might not involve $j_{\ell}$ or $i_{h}$.

Initially, we have no windows. If at time $t, i_{h}$ is not "in a window" then a window $\left[x_{t}\left(i_{h}\right), x_{t}\left(i_{h}\right)+m_{0}+m_{1}\right]$ is created at time $t$. If (i) $i_{h}$ has an associated 
$\eta$-value $2^{*}$ at time $t$; (ii) in interval $\left[x_{t}\left(i_{h}\right), x_{t}\left(i_{h}\right)+m_{0}+m_{1}\right]$ is a $\xi$-particle $j_{\ell}$ associated with a $\eta$-particle of type $4^{*}$.

A window $\left[x_{t_{0}}\left(i_{h}\right), x_{t_{0}}\left(i_{h}\right)+m_{0}+m_{1}\right]$ will survive until (a) there is a joining (inside the interval) of a $\xi$-particle with a type $2^{*}$ (or lower) $\eta$-particle, or the coupled exclusion process $(\xi, \eta)$ evolves so that such a joining is impossible on $\left[x_{t_{0}}\left(i_{h}\right), x_{t_{0}}\left(i_{h}\right)+m_{0}+m_{1}\right]$ without particles entering or leaving the window; (b) A $\xi$ - or $\eta$-particle on $\left(x_{t_{0}}\left(i_{h}\right)+m_{1}+m_{0}, \infty\right)$ tries to jump to a site in $\left(-\infty, x_{t}\left(i_{h}\right)+m_{1}+m_{0}\right]$ or a $\xi$ - or $\eta$-particle in $\left(-\infty, x_{t_{0}}\left(i_{h}\right)\right)$ tries to jump to a site in $\left[x_{t_{0}}\left(i_{h}\right), \infty\right)$.

The various windows in existence at a given time may overlap. Thus when there is a joining of a $\xi$-particle with a $\eta$-type $2^{*}$ (or lower) particle, it may be simultaneously occurring within (at most $m_{0}+m_{1}$ ) many windows. We wish to credit this coupling to a marked $\xi$-vacancy $i_{h}$. To do this we randomly choose among the windows having a claim, and then, given our window, we randomly choose an $i_{h}$ to credit among the (again as most $m_{0}+m_{1}$ ) eligible $i_{h}$ 's in that window.

We define $W\left(i_{h}\right)$ (a lower bound on the number of windows that $i_{h}$ has been in) as follows.

Let

$$
\begin{aligned}
& S_{0}^{h}=0, \\
& T_{1}^{h}=\inf \left\{t \geq S_{0}^{h}:\left(i_{h} \text { is in a window }\right) .\right.
\end{aligned}
$$

For $v \geq 1$,

$$
\begin{aligned}
& S_{v}^{h}=\inf \left\{t>T_{v}^{h}: \text { the window }\left[x_{T_{v}^{h}}, x_{T_{v}^{h}}+m_{0}+m_{1}\right] \text { dies }\right\}, \\
& T_{v}^{h}=\inf \left\{t>S_{v-1}^{h}:\left(i_{h} \text { is in a window }\right)\right\} .
\end{aligned}
$$

Of course, often $T_{v}^{h}=S_{v-1}^{h}$.

Definition. $W\left(i_{h}\right)=\sup \left\{v: S_{v}^{h} \leq k N\right\}$.

The idea is that a typical $i_{h}$ cannot pass through or past many $j_{\ell}$ without $W$ becoming large and that $W$ cannot become large without $i_{h}$ becoming credited.

LEMMA 5.1. For some $c$ (depending on $m_{0}, m_{1}$ but not on $N$ ) we have [uniformly over all $(\xi, \eta)_{T}$ ] and $i_{h}, P\left[W\left(i_{h}\right)>R, i_{h}\right.$ is not credited] $\leq(1-c)^{R}$.

Proof. This simply follows from the Markov property and the fact that there are only finitely possible $(\xi, \eta)$ configurations on $\left(\{0,1\}^{\left[0, m_{0}+m_{1}\right]}\right)^{2}$.

Proof of Theorem. Let $B$ be the event that in time $[T, k N]$ on interval $\left[-\left(2 C N+m_{1}+m_{0}\right), 2 C N+m_{1}+m_{0}\right]$ at least $\frac{\varepsilon \delta}{20} N \xi$-particles change their $\eta$-class to 3 or lower or at least $\frac{\varepsilon \delta}{20} N \xi$-vacancies change their class to 3 or higher. 
Obviously, if $P(B)>1 / 3$ (say) then we have that [by ergodicity of $(\xi, \eta)_{T}$ ] the density of $\xi$-particles taking $\eta$-value in $[0, a+\delta]$ or in $[0, b]$ will increase by at least $1 / 6 \frac{\varepsilon \delta}{20\left(4 C+\frac{2\left(m_{1}+m_{0}\right)}{N}\right)} \gg \varepsilon^{\prime}$ which will contradict our definition of $T$, and thereby the assumption that $M_{t} \nrightarrow U[0, \alpha]$.

We consider the following "bad" events:

1. The number of $2^{*} \eta$-particles that are $\xi$-vacant is less than $M=N\left(\varepsilon \delta-\varepsilon^{\prime}\right)$ or the number of $4^{*} \eta$-particles that are $\xi$-occupied is less than $M$.

2. The number of $i_{h}, 1 \leq h \leq M$ so that $X\left(i_{h}\right) \geq \varepsilon^{\prime 3 / 2} N$ is $\geq \varepsilon^{\prime} N$ or the number of $j_{l}, 1 \leq l \leq M$ so that $X\left(j_{l}\right) \geq \varepsilon^{\prime 3 / 2} N$ is greater than or equal to $\varepsilon^{\prime} N$.

3. The number of $i_{h}$ or $j_{\ell}$ that leave [ $\left.-2 C N, 2 C N\right]$ in time interval [T, $\left.k N\right]$ is greater than or equal to $\varepsilon^{\prime} N$.

4. The conclusion of Proposition 3.3 does not hold with $\gamma=\left(\varepsilon^{\prime}\right)^{2} / 10^{10}$.

5. At least one $i_{h}$ has the property that $i_{h}$ is associated with a $\eta-2^{*}$ particle as time $k N, i_{h}$ is not credited and $W\left(i_{h}\right) \geq \frac{\varepsilon \delta N}{5\left(m_{1}+2 m_{0}\right)^{2}}$.

By lower bounds for $N, D$, and $E$ and Proposition 3.3, we have that the probability of $(1) \rightarrow$ (4) occurring is bounded by $1 / 10$. By Lemma 5.1 the probability of (5) is bounded by $N(1-c)^{\varepsilon \delta N / 5\left(m_{1}+2 m_{0}\right)^{2}}<1 / 10$ if $N$ is sufficiently large (as we will take $N$ to be).

So with probability $1-1 / 10-1 / 10=8 / 10$ (at least) we have that $(1) \rightarrow$ (5) do not occur. We claim that in this case event $B$ must occur. If not, then by $(1)^{c}$ there are $M$ labelled $\eta$ - $2^{*} \xi$-vacancies $i_{h}$ in $[0, N]$ and $M$ labelled $\eta-4^{*} \xi$-particles $j_{l}$ in $\left[N+m_{0}, 2 N\right]$ at time $T$.

By $(3)^{c}$ and $B^{c}$ occurring we have that for at least $\left(M-\varepsilon^{\prime} N-\frac{N \varepsilon \delta}{20}\right) h$ in $\{1, \ldots, M\}$ and $\left(M-\varepsilon^{\prime} N-\frac{N \varepsilon \delta}{20}\right) \ell$ in $\{1, \ldots, M\} i_{h}, j_{\ell}$ remains inside $[-2 C N,+$ $2 C N\}$ in time interval $[T, k N]$ and $i_{h}$ retains a $\eta-2^{*}$ value, $j_{\ell}$ an $\eta-4^{*}$ value throughout.

By $(4)^{c}$ of these at least $M-\varepsilon^{\prime} N-\frac{N \varepsilon \delta}{20}-\varepsilon^{\prime} N i_{h}$ 's will be to the right of $M-\varepsilon^{\prime} N-\frac{N \varepsilon \delta}{20}-\varepsilon^{\prime} N$ of the $j_{\ell}$ 's. Let the two indexes be

$$
\begin{array}{ll}
I \subseteq\{1, \ldots, M\}, & |I| \geq M-\varepsilon^{\prime} N-\frac{N \varepsilon \delta}{20}-\varepsilon^{\prime} N, \\
J=\{1, \ldots, M\}, & |J| \geq M-\varepsilon^{\prime} N-\frac{N \varepsilon \delta}{20}-\varepsilon^{\prime} N .
\end{array}
$$

By $(2)^{c}$ we finally have subsets

$$
\begin{gathered}
I^{\prime} \subseteq\{1, \ldots, M\}, \quad J^{\prime} \leq\{1, \ldots, M\}, \\
\left|I^{\prime}\right|,\left|J^{\prime}\right| \geq M-\varepsilon^{\prime} N-\frac{N \varepsilon \delta}{20}-\varepsilon^{\prime} N-\varepsilon^{\prime} N \geq \frac{8 N \varepsilon \delta}{10} .
\end{gathered}
$$

Taking subsets if necessary we suppose $\left|I^{\prime}\right|,\left|J^{\prime}\right|=\frac{8 N \varepsilon \delta}{10}$. For $N$ large so that $h \in I^{\prime}, \ell \in J^{\prime}:$ 
(a) $x_{t}\left(i_{h}\right), x_{t}\left(j_{\ell}\right) \in[-2 C N, 2 C N] \forall t \in[T, k N]$.

(b) $x_{k N}\left(i_{h}\right)>x_{k N}\left(j_{\ell}\right)$.

(c) At time $k N, i_{h}$ has $\eta$-value $2^{*}$ and $j_{\ell}$ has value $4^{*}$.

(d) $X\left(i_{h}\right) \leq \varepsilon^{\prime 3 / 2} N, X\left(j_{\ell}\right) \leq \varepsilon^{\prime 3 / 2} N$.

For $\ell \in J^{\prime}$, let $D_{\ell}=\left\{h\right.$ : for some $t \in[T, k N], x_{t}\left(j_{\ell}\right)-m_{0}<x_{t}\left(i_{h}\right)<$ $\left.x_{t-}\left(j_{\ell}\right)-\left(m_{1}+m_{0}\right)\right\}$.

By hypothesis, $\forall \ell \in J^{\prime}\left|D_{\ell}\right| \leq{\varepsilon^{\prime 3 / 2}}^{3 / 2}$ so $\Sigma_{\ell \in J^{\prime}}\left|D_{\ell}\right| \leq \varepsilon^{\prime 3 / 2} N \frac{8 N \varepsilon \delta}{10}$. So by Chebyshev number of $h \in I^{\prime}: \Sigma_{\ell \in J^{\prime}} I_{h \in D_{\ell}} \geq \varepsilon^{\prime} N$ is less than or equal to $N \varepsilon \delta \varepsilon^{\prime 1 / 2}$. So let $I^{\prime \prime}=\left\{h \in I^{\prime}: \Sigma_{\ell \in J^{\prime}} I_{h \in D_{\ell}} \leq \varepsilon^{\prime} N\right\}$; then $\left|I^{\prime \prime}\right| \geq \frac{7}{10} N \varepsilon \delta$.

As noted, if (b) occurs for $h \in I^{\prime \prime}, \ell \in J^{\prime}$ then either for some $t \in[T, k N]$,

(A) $x_{t}\left(i_{h}\right)+m_{0} \leq x_{t}\left(j_{\ell}\right) \leq x_{t}\left(i_{h}\right)+m_{0}+m_{1}$;

(B) $x_{t}\left(j_{\ell}\right)-m_{0}<x_{t}\left(i_{h}\right)<x_{t-}\left(j_{\ell}\right)-\left(m_{1}+m_{0}\right)$;

(C) $x_{t}\left(i_{h}\right)+m_{0}+m_{1}<x_{t}\left(j_{\ell}\right)<x_{t}\left(i_{h}\right)+m_{0}$.

By property (d) of $I^{\prime}$ and therefore of $I^{\prime \prime}$, the number of $\ell$ such that (C) holds is bounded by $\varepsilon^{\prime 3 / 2} N$; by definition of $I^{\prime \prime}$ the number of $\ell$ so that (B) holds is bounded by $\varepsilon^{\prime} N$, so for $h \in I^{\prime \prime}$ at least $\left|J^{\prime}\right|-\varepsilon^{\prime} N-\varepsilon^{\prime 3 / 2} N \geq \frac{7 N \varepsilon \delta}{10} \ell$ satisfy (A).

We denote this subset of $J^{\prime}$ by $G_{h}$. By definition of window, for every $S_{i}^{h}$ at most $m_{1}+2 m_{0} j_{\ell}$ in $G_{h}$ can pass from $m_{1}+m_{0}$ to the right of $i_{h}$ to less than $m_{0}$ to the right. Thus it must be the case that for $h \in I^{\prime \prime} W\left(i_{h}\right) \geq \frac{7 N \varepsilon \delta}{10} / m_{1}+2 m_{0}$.

Thus by (5) we must have $i_{h}$ is credited. $\forall h \in I^{\prime \prime}$ but as $x_{t}\left(i_{h}\right) \in[-2 C N$, $2 C N] \forall t \in[T, k N]$. This means that at least $\left(I^{\prime \prime}\right) \xi$-particles decreased their $\eta$-value to $2^{*}$ or lower in $\left[-2 C N-\left(m_{0}+m_{1}\right) 2 C N+m_{0}+m_{1}\right]$; that is, $B$ occurs.

Acknowledgments. This paper owes much to a discussion with Enrique Andjel in which he explained some comments of Bramson. I am happy to acknowledge my debt to an extremely diligent referee who has greatly improved the clarity of this paper.

\section{REFERENCES}

[1] ANDJEL, E. (1981). The asymmetric simple exclusion process on $Z^{d}$. Z. Warsch. Verw. Gebiete 58 423-432.

[2] Ekhaus, M. and Gray, L. (2000). A strong law for the motion of interfaces in particle systems. Preprint.

[3] Evans, L. C. (1998). Partial Differential Equations. Amer. Math. Soc., Providence, RI.

[4] Ferrari, P. A. (1992). Shock fluctuations in the asymmetric simple exclusion process. Probab. Theory Related Fields 91 81-101.

[5] Ferrari, P. A. Kipnis, C and SAAda, E. (1991). Microscopic structure of travelling waves in the asymmetric simple exclusion process. Ann. Probab. 19 226-244.

[6] KIPNIS, C. (1986). Central limit theorems for infinite systems of queues and applications to simple exclusion. Ann. Probab. 14 397-408.

[7] Kipnis, C. and Landim, C. (1999). Hydrodynamical Limits of Particle Systems. Springer, Berlin.

[8] Liggett, T. M. (1985). Interacting Particle Systems. Springer, Berlin.

[9] Liggett, T. M. (1999). Developments Some Particle Systems: Contact, Voter and Exclusion Processes. Birkhäuser, Boston. 
[10] Mountford, T. and Prabhakar, B. (1995). On the weak convergence of departures from an infinite series of $\cdot / M / 1$ queues. Ann. Appl. Probab. 5 121-127.

[11] Rezakhanlou, F. (1991). Hydrodynamic limit for attractive particle systems on $Z^{d}$. Comm. Math. Phys. 140 417-448.

DEPARTMENT OF Mathematics

UNIVERSITY OF CALIFORNIA

LOS ANGELES, CALIFORNIA 90095-1555 